\title{
Differential Equations for Scaling Relation in $N=2$ Supersymmetric SU(2) Yang-Mills Theory Coupled with Massive Hypermultiplet
}

\author{
Yüji Ohta \\ Research Institute for Mathematical Sciences \\ Kyoto University \\ Sakyoku, Kyoto 606, Japan.
}

\begin{abstract}
Differential equations for scaling relation of prepotential in $N=2$ supersymmetric SU(2) Yang-Mills theory coupled with massive matter hypermultiplet are proposed and are explicitly demonstrated in one flavour $\left(N_{f}=1\right)$ theory. By applying Whitham dynamics, the first order derivative of the prepotential over the $T_{0}$ variable corresponding to the mass of the hypermultiplet, which has a line integral representation, is found to satisfy a differential equation. As the result, the closed form of this derivative can be obtained by solving this equation. In this way, the scaling relation of massive prepotential is established. Furthermore, as an application of another differential equation for the massive scaling relation, the massive prepotential in strong coupling region is derived.
\end{abstract}

PACS: 11.15.Tk, 11.15.Me, 12.60.Jv, 02.30.Hq. 


\section{INTRODUCTION}

It is well-known that the low energy effective action of $N=2$ supersymmetric YangMills theory is described in terms of holomorphic prepotential $\mathcal{F}$. In this case, the perturbative part of the prepotential is not modified beyond one-loop order according to the non-renormalization theorem, 胜国 but is known to be affected by instantons. In the case of $\mathrm{SU}(2)$ gauge group, Seiberg and Witten䀦 proposed a general prescription to determine the non-perturbative prepotential with the aid of Riemann surface of genus one. Based on their observation, Klemm et al. $\mathrm{\text {O }}$ determined the instanton corrected prepotential by adopting a method of Picard-Fuchs equation, which was often used in mirror symmetry of Calabi-Yau manifold 0.10 .11 On the other hand, Matond 2 derived a recurrence formula of the instanton expansion coefficients of the prepotential by noticing a modularity. As a bonus, he obtained a quite simple relation between prepotential and moduli often referred as scaling relation. After this discovery, the existence of such relation in $N=2$ supersymmetric Yang-Mills theory coupled with or without massive quark hypermultiplets was pointed out independently by two groups 13.14 Sonnenschein et al. 13 proved it by noticing the homogeneity of the prepotential, while Eguchi and Yang 14 established the same result (2.17) in the language of Whitham dynamics. 5 . 1 . However, in the framework of Whitham dynamics, we always encounter the problem of evaluating the derivative of $\mathcal{F}$ over the $T_{0}$ variable in order to establish a scaling relation when the mass of the hypermultiplets is not ignored. In the Whitham theory, this quantity is represented by a line integral interpolating two coverings of Riemann surface and therefore its evaluation is quite complicated, especially, in massive case. In fact, explicit calculation of this integral is not found in literatures.

One of the aim of the paper is to give a solution to this problem. However, since the calculation in the case of a theory with $N_{f}$ massive flavours is very complicated even for $\mathrm{SU}(2)$, we treat only $\mathrm{SU}(2) N_{f}=1$ case. In addition, extension to $N_{f}>1$ is straightforward, so the reader is recommended to try to proceed other cases. Our construction starts from comparing Wronskian of massive Picard-Fuchs equation with the modular invariant of Matone.12 These two are shown to be related by a Fuchsian differential equation. Applying Whitham theory of soliton to this equation, we can obtain a differential equation for $\partial \mathcal{F} / \partial T_{0}$. In this 
way, it is determined as a solution to this equation. The details are discussed in Sec. II. On the other hand, in the course of the calculus used in the derivation of this differential equation, we can find another simple differential equation, which indicates a relation between

prepotential and moduli like that in five dimensions.17 This equation is also a consequence of scaling relation in the massive theory. As an application, the prepotential in strong coupling region (dual prepotential) is derived in Sec. III. At first sight, the dual prepotential looks very complicated, but the massless limit coincides with that in the massless theory obtained by Ito and Yang. 18 Sec. IV is a brief summary.

\section{SCALING RELATION AND WHITHAM HIERARCHY}

\section{A. The Picard-Fuchs equation}

First of all, let us recall the basics of the massive $N_{f}=1$ theory in Seiberg-Witten approach. In the case of $\mathrm{SU}(2)$, we can take two kinds of curves, one of which is elliptic type 7 and the other is hyperelliptic type 19 Though an elliptic curve is used in the next section, here we take the hyperelliptic curve. In this case, it is given by

$$
y^{2}=\left(x^{2}-u\right)^{2}-\Lambda^{3}(x+m),
$$

where $u=\left\langle\operatorname{tr} \phi^{2}\right\rangle$ is the moduli ( $\phi$ is the complex scalar field of $N=2$ chiral superfield), $m$ is the mass of the hypermultiplet and $\Lambda$ is the mass scale parameter of this $N_{f}=1$ theory. For this curve, the Seiberg-Witten differential 1-form $\lambda$ is determined from the basic relation $d \lambda / d u \propto d x / y$. Taking into account of the numerical normalization factor, we find 19,20

$$
\lambda=\frac{\sqrt{2}}{4 \pi i} \frac{x d x}{y}\left[\frac{x^{2}-u}{2(x+m)}-2 x\right] .
$$

In general, Seiberg-Witten 1-form in a theory involving massive matter hypermultiplets in the fundamental representation of the gauge group is endowed with pole structure whose residue is linearly proportional to the masses of the hypermultiplets.0.19

Seiberg-Witten ansatz requires that the vacuum expectation value of $\phi$ and its dual are quantum mechanically given by the two periods 


$$
a=\oint_{\alpha} \lambda, a_{D}=\oint_{\beta} \lambda
$$

respectively, along the canonical basis $(\alpha \cap \beta=+1)$ of 1 -cycles on (2.1). Then the periods satisfies the Picard-Fuchs equation

$$
\frac{d^{3} \Pi}{d u^{3}}+X \frac{d^{2} \Pi}{d u^{2}}+Y \frac{d \Pi}{d u}=0
$$

where

$$
\begin{aligned}
& X=\frac{d}{d u} \ln \frac{\Delta}{4 m^{2}-3 u}, \\
& Y=-\frac{8}{\Delta}\left[4\left(2 m^{2}-3 u\right)+3 \frac{3 m \Lambda^{3}-4 u^{2}}{4 m^{2}-3 u}\right] .
\end{aligned}
$$

Here,

$$
\Delta(u)=256 u^{3}-256 m^{2} u^{2}-288 m \Lambda^{3} u+256 m^{3} \Lambda^{3}+27 \Lambda^{6}
$$

is the discriminant of the curve (2.1).

\section{B. Differential equations for scaling relation}

Differential equation for Wronskian can be used to make a relation to prepotential. The reader might know the example in the context of mirror symmetry presented by Candelas et al.,10,11 who used "Wronskian" in order to make a contact with Yukawa coupling in complex structure moduli space. Similar presentation is possible also in $N=2$ supersymmetric Yang-Mills theory coupled with or without massless matter hypermultiplets. In the case of SU(2), integral of Wronskian of Picard-Fuchs equation yields the scaling relation

of prepotential. 14 However, when hypermultiplets are massive, it is not easy to see such a simple relation connecting prepotential and moduli. We encountered in a similar problem in the five dimensional gauge theory.17 Since we did not know a five dimensional analogue of the scaling relation in four dimensional gauge theory, we proposed a differential relation between prepotential and Wronskian. The method used in the course of this calculation reveals further aspects on scaling relation, provided it is applied to four dimensional gauge theory.

For this, let us prepare the following two quantities 


$$
W=a^{\prime} a_{D}^{\prime \prime}-a^{\prime \prime} a_{D}^{\prime}, w=a a_{D}^{\prime}-a^{\prime} a_{D},
$$

where $^{\prime}=d / d u$. The first equation is precisely the Wronskian for the third order PicardFuchs equation (2.4), while the integration of the second one produces the modular invariant of Matone 12

$$
\int_{0} w d u=a a_{D}-2 \mathcal{F}
$$

Remark: Modular invariance mentioned here is the sense of pure SU(2) theory. Here, the integral symbol indicates that it is an integration constant free integral, namely, the integration constant is set to zero. What we would like to clarify is a relation between (2.8) and (integration of) $W$, so the first task is to try to connect $W$ and $w$.

Fortunately, this is simply done by differentiating $w$ over $u$ repeatedly modulo the PicardFuchs equation (2.4), and we can find

$$
w^{\prime \prime}+X w^{\prime}+Y w=W
$$

It is interesting to notice that $w$ satisfies a Picard-Fuchs equation with $W$ as a source term. On the other hand, for $W$ we can easily obtain

$$
W^{\prime}+X W=0
$$

which implies

$$
W=c \frac{4 m^{2}-3 u}{\Delta}
$$

where $c$ is an integration constant. Note that the discriminant of the curve appears in the denominator and a similar relation was noticed in five dimensions. 17 In order to fix $c$, we may use massless or double scaling limit as a boundary condition. For example, in the massless limit, it is well-known that $w=i 3 /(4 \pi), 21$ therefore, from $(2.9)$

$$
c=-i \frac{16}{\pi}
$$

Of course, $c$ must be uniquely fixed, and the same value is obtained from double scaling limit. (2.11) with (2.12) plays an important role in the next section.

In this way, we arrive at 


$$
w^{\prime \prime}+X w^{\prime}+Y w=-i \frac{16}{\pi} \frac{4 m^{2}-3 u}{\Delta}
$$

Since the solution to this equation gives $w$ and must be related with (2.8), $w$ obtained from (2.13) is expected to give a scaling relation in this massive theory 13

$$
a \frac{\partial \mathcal{F}}{\partial a}-2 \mathcal{F}=-m \frac{\partial \mathcal{F}}{\partial m}-\Lambda \frac{\partial \mathcal{F}}{\partial \Lambda}
$$

where $\mathcal{F}$ is regarded here as a homogeneous function $\mathcal{F}=\mathcal{F}(a, \Lambda, m)$ in variables. Specifically, the solution of (2.13) will include informations on the right hand side of (2.14) and what we would like to do next is to extract such informations from (2.13). The best way to accomplish this is to introduce the Whitham theory.15.16

\section{Relation to Whitham dynamics}

Let us consider a consequence of (2.13) in view of Whitham dynamics in $N=2$ YangMills theory. For details of Whitham dynamics in the context of Yang-Mills theory, see Ref.14-16.

Let $T_{n}(n \in N \cup\{0\})$ be time variables coupled to $(n+1)$-th order pole of SeibergWitten differential 1-form. In the $\mathrm{SU}(2) N_{f}=1$ theory, the prepotential is available from the relation

$$
\frac{\partial \mathcal{F}}{\partial a}=\oint_{\beta} \lambda, \frac{\partial \mathcal{F}}{\partial T_{n}}=-2 \pi i \operatorname{res}\left(z^{-n} \lambda\right), \frac{\partial \mathcal{F}}{\partial T_{0}}=-2 \pi i \int_{z_{*}=-m}^{z=-m} \lambda
$$

where residue is evaluated at $x=1 / z=\infty$ and $z_{*}(=1 / x)$ is the coordinate on the other sheet of the curve. At $x=\infty, \lambda$ is expanded as

$$
\lambda=\left[-\sum_{n>0}^{\infty} n T_{n} z^{-n-1}+T_{0} z^{-1}-\frac{1}{2 \pi i} \sum_{n>0}^{\infty} \frac{\partial \mathcal{F}}{\partial T_{n}} z^{n-1}\right] d z
$$

Then, Whitham dynamics in $N=2$ Yang-Mills theory implies the homogeneity relation of the prepotential 14

$$
a \frac{\partial \mathcal{F}}{\partial a}-2 \mathcal{F}=-T_{0} \frac{\partial \mathcal{F}}{\partial T_{0}}-T_{1} \frac{\partial \mathcal{F}}{\partial T_{1}}
$$

Note that the right hand side of (2.17) should be identified with that in (2.14).

In the framework of Whitham hierarchy, $T_{0}, T_{1}$ and $\partial \mathcal{F} / \partial T_{1}$ are read from (2.16) as 


$$
T_{0}=-i \frac{m}{4 \sqrt{2} \pi}, T_{1}=i \frac{3}{4 \sqrt{2} \pi}, \frac{\partial \mathcal{F}}{\partial T_{1}}=\sqrt{2}\left(\frac{m^{2}}{4}-u\right)
$$

Due to our normalization, the numerical factors are different from those used by Eguchi and Yang.14 Note that $T_{0}$ vanishes when $m=0$. This simplifies (2.17) and because of this, the scaling relation in the massless theory is easily determined by using Whitham hierarchy. In this case, it is not necessary to know $\partial \mathcal{F} / \partial T_{0}$, but in massive case it must be known. However, in a massive theory the calculation of $\partial \mathcal{F} / \partial T_{0}$ requires a care because the third equation in (2.15) is a line integral from one covering of the Riemann surface to the other. In the case at hand, this integral consists of two pieces of integral from $-m$ to $\infty$ on one sheet and a copy of it on the other sheet (when the mass vanishes, it reduces to a familiar integral 4 ). Due to the pole of $\lambda$ at $x=-m$, the evaluation of it is not easy and it is therefore still on challenging stage. However, we can develop another method and show that $\partial \mathcal{F} / \partial T_{0}$ satisfies a differential equation with the aid of (2.13). Then $\partial \mathcal{F} / \partial T_{0}$ is obtained as a solution to this equation.

Differentiation of (the left hand side of) (2.17) gives $w$, so a differential equation for $\partial \mathcal{F} / \partial T_{0}$ can be obtained by substituting $w$ calculated from (2.17) with (2.18) into (2.13)

$$
\Theta^{\prime \prime \prime}+X \Theta^{\prime \prime}+Y \Theta^{\prime}=\frac{4 \sqrt{2}}{m}\left[i \frac{\pi}{2} W+\frac{6}{\Delta}\left(-8 m^{2}+3 \frac{3 m \Lambda^{3}-4 u^{2}}{4 m^{2}-3 u}\right)\right],
$$

where $\Theta=\partial \mathcal{F} / \partial T_{0}$. It is easy to get a solution to this equation

$$
\Theta^{\prime}=c_{1} a^{\prime}+c_{2} a_{D}^{\prime}+i \frac{2 \sqrt{2} \pi}{m} w+i \frac{3 \sqrt{2} \pi}{2 m}\left[a_{D}^{\prime} \int_{0} \frac{a^{\prime} Z}{4 m^{2}-3 u} d u-a^{\prime} \int_{0} \frac{a_{D}^{\prime} Z}{4 m^{2}-3 u} d u\right]
$$

where $c_{i}$ are integration constants and

$$
Z=-8 m^{2}+3 \frac{3 m \Lambda^{3}-4 u^{2}}{4 m^{2}-3 u}
$$

In the derivation of (2.20), we have used the fact that a second order differential equation

$$
\frac{d^{2} y}{d x^{2}}+P(x) \frac{d y}{d x}+Q(x) y=R(x)
$$

with any function $P(x), Q(x)$ and $R(x)$ has a general solution in the form

$$
y=c_{1} y_{1}+c_{2} y_{2}-y_{1} \int_{0} \frac{y_{2} R(x)}{W\left(y_{1}, y_{2}\right)} d x+y_{2} \int_{0} \frac{y_{1} R(x)}{W\left(y_{1}, y_{2}\right)} d x
$$


where $y_{i}$ are two independent solutions in the case of $R(x)=0$ and $W\left(y_{1}, y_{2}\right)$ is its Wronskian. Since (2.19) without the right hand side is nothing but the massive Picard-Fuchs equation, the above $y_{i}$ may be chosen as $a^{\prime}$ and $a_{D}^{\prime}$. Then $W\left(y_{1}, y_{2}\right)$ is identified with $W$ defined in (2.7). Furthermore, (2.11) is used to arrive at the final expression (2.20).

Let us see the massless limit of (2.20). In this limit, naively, the factor $1 / m$ diverges, but we leave it for the moment. When $m$ vanishes, the integrals can be easily evaluated and the resulting terms cancels out the third term in (2.20). Therefore, it follows that

$$
\left.\frac{\partial \mathcal{F}}{\partial T_{0}}\right|_{m \rightarrow 0}=c_{1} a+c_{2} a_{D}+\text { const. }
$$

This result reflects the fact that (2.19) reduces to the total differentiation of the massless Picard-Fuchs equation because of vanishing of the right hand side of (2.19) for $m \rightarrow 0$. Actually, $\partial \mathcal{F} / \partial T_{0}$ corresponds to $\partial \mathcal{F} / \partial m$, so all constants in (2.24) should be zero for $m \rightarrow 0$.

On the other hand, integrating (2.20) for $m \neq 0$, we obtain

$$
a a_{D}-2 \mathcal{F}=i \frac{m}{6 \sqrt{2} \pi}\left(c_{1} a+c_{2} a_{D}\right)+c_{3}-\frac{1}{4} \int_{0}\left[a_{D}^{\prime} \int_{0} \frac{a^{\prime} Z}{4 m^{2}-3 u} d u-a^{\prime} \int_{0} \frac{a_{D}^{\prime} Z}{4 m^{2}-3 u} d u\right] d u
$$

where $c_{3}$ is an integration constant, with the aid of (2.17) and (2.18). This is the general form of massive scaling relation.

Remark: If the prepotential is known,20 $c_{i}$ are easily determined as

$$
c_{1}=-3 \pi i n^{\prime}, c_{2}=3 \pi i n, c_{3}=-\frac{m^{2}}{16}\left(i+4 i \ln 2-2 \pi n n^{\prime}\right),
$$

where $n, n^{\prime} \in \boldsymbol{Z}$ are the winding numbers of 1-cycles around the pole corresponding to $x=$ $-m$ of $\lambda$. These constants supports the massless limit behaviour of $\partial \mathcal{F} / \partial T_{0}$. However, precisely, these integration constants must be determined from comparing it with the result of lower order expansion of the third equation in (2.15).

\section{MASSIVE PREPOTENTIAL IN STRONG COUPLING REGIME}

Next, let us study the prepotential in the strong coupling region following to the technology recently developed in the five dimensional gauge theory.17 The basic tool in our case is 2.11$)$. 
For later convenience, we take the elliptic curve 7

$$
y^{2}=x^{2}(x-u)+\frac{1}{4} m \Lambda^{3} x-\frac{\Lambda^{6}}{64}
$$

whose discriminant coincides with (2.6). The Seiberg-Witten differential 1-form is given by

$$
\lambda=\frac{\sqrt{2}}{8 \pi} \frac{d x}{y}\left[2 u-3 x-\frac{m \Lambda^{3}}{4 x}\right]
$$

For this curve, we choose the 1-cycles on the surface (3.1) as Ito-Yang cycles, 18 which reduce for large $u$ with vanishing mass

$$
\alpha:-i \frac{\Lambda^{3}}{8 \sqrt{u}} \longrightarrow+i \frac{\Lambda^{3}}{8 \sqrt{u}}, \beta: u \longrightarrow-i \frac{\Lambda^{3}}{8 \sqrt{u}} .
$$

Also in this case, the periods are defined by (2.3) and satisfy (2.4).

To find strong coupling regime, let us decompose the discriminant as

$$
\Delta(u)=256 \prod_{i=1}^{3}\left(u-e_{i}\right)
$$

where $e_{i}$ are given by the vanishing points of $\Delta(u)=0$, which correspond to the strong coupling regime. $e_{i}$ can be easily obtained by solving $\Delta(u)=0$ for $u$, but we should take care of the derivation. In general, any cubic equation in $x$

$$
x^{3}+a x^{2}+b x+c=0,
$$

where $a, b$ and $c$ are some constants independent of $x$, has three independent solutions. One of them is

$$
x=-\frac{2^{1 / 3}\left(-a^{2}+3 b\right)}{3\left[-2 a^{3}+9 a b-27 c+\sqrt{4\left(-a^{2}+3 b\right)^{3}+\left(-2 a^{3}+9 a b-27 c\right)^{2}}\right]^{1 / 3}}+\cdots,
$$

where $\cdots$ means omission of remaining terms. However, if the denominator vanishes

$$
-2 a^{3}+9 a b-27 c+\sqrt{4\left(-a^{2}+3 b\right)^{3}+\left(-2 a^{3}+9 a b-27 c\right)^{2}}=0,
$$

i.e.,

$$
\left(a^{2}-3 b\right)^{3}=0
$$

the formula (3.6) is not valid any more. In this case, one must start again from (3.5) under the condition (3.8). 
In the case at hand, the condition (3.8) for (2.6) corresponds to

$$
m^{3}(2 m+3 \Lambda)^{3}\left(4 m^{2}-6 m \Lambda+9 \Lambda^{2}\right)^{3}=0
$$

which implies that the directly obtained location under $m \neq 0$ (c.f. (3.6)), does not reflect precise massless limit of the massive discriminant. To understand more illustratively, let us set $u=x+i y$, where $x, y \in \boldsymbol{R}$. Then the equation $\Delta(u)=0$ produces $3\left(x-m^{2} / 3\right)^{2}-y^{2}=$ $m\left(8 m^{3}+27\right) / 24$. For a non-zero $m$, this is nothing but hyperbolic curves, but for $m=0$ it becomes to crossing lines. Clearly, the zeros of the discriminant at $m=0$ behave singular because transition from hyperbolic curve to lines is always suppressed.

In fact, though the correct massless location in the strong coupling regime must be

$$
e_{1}^{(0)}=-\frac{3 \Lambda^{2}}{2^{8 / 3}}, e_{2}^{(0)}=\frac{3(1-i \sqrt{3}) \Lambda^{2}}{2^{11 / 3}}, e_{3}^{(0)}=\frac{3(1+i \sqrt{3}) \Lambda^{2}}{2^{11 / 3}}
$$

these can not be obtained from (3.6) with $m=0$. Therefore, (3.9) distinguishes regions in quantum moduli space at $m=0$ and $m \neq 0$. To see a connection with massless theory, for example, we realize the zero locus of massive discriminant as a small mass perturbation in the form $e_{i}=e_{i}^{(0)}+($ series in $m)$, where this series converges for $|m|<1$. Substituting this into the equation $\Delta=0$ and equating coefficient of powers in $m$ to zero, one finds

$$
\begin{aligned}
& e_{1}=e_{1}^{(0)}-\frac{m}{2^{1 / 3}} \Lambda+\frac{m^{2}}{3}-\frac{4}{27} \frac{2^{1 / 3} m^{3}}{\Lambda}+\frac{4}{81} \frac{2^{2 / 3} m^{4}}{\Lambda^{2}}+\cdots \\
& e_{2}=e_{2}^{(0)}-\frac{(1+i \sqrt{3})}{2^{4 / 3}} m \Lambda+\frac{m^{2}}{3}+\frac{2^{4 / 3}(1-i \sqrt{3}) m^{3}}{27 \Lambda}+\cdots \\
& e_{3}=e_{3}^{(0)}+\frac{(1-i \sqrt{3})}{2^{4 / 3}} m \Lambda+\frac{m^{2}}{3}+\frac{2^{4 / 3}(1+i \sqrt{3}) m^{3}}{27 \Lambda}+\cdots
\end{aligned}
$$

As a final check, (3.4) must be satisfied, but this can be easily confirmed by order by order in $m$ greater than $m^{3}$. Note that (3.11) coincides with the massless strong coupling points for $m=0$ and thus the above $e_{i}$ are the expected ones for small but finite mass. Below, to make a contact with the result of Ito and Yang,18 $e_{1}$ is chosen as a representative of strong coupling regime. On the other hand, for $m$ greater than or equal to 1, (3.6) is available to derive zeros of the discriminant.

Remark: In the case of $S U(2)$ gauge group, this situation is characteristic to the $N_{f}=1$ theory. For $N_{f}=2$ and 3 theories' discriminants, we do not encounter with such sensitive problem in the determination of strong coupling region. 
Performing differential calculation between periods and dual prepotential

$$
\begin{aligned}
& \frac{d a}{d u}=\frac{\mathcal{F}_{D}^{\prime \prime}}{u^{\prime}}, \\
& \frac{d^{2} a}{d u^{2}}=\frac{1}{u^{\prime 3}}\left(\mathcal{F}_{D}^{\prime \prime \prime} u^{\prime}-\mathcal{F}_{D}^{\prime \prime} u^{\prime \prime}\right), \\
& \frac{d a^{3}}{d u^{3}}=\frac{1}{u^{\prime 5}}\left[\left(\mathcal{F}_{D}^{(4)} u^{\prime}-\mathcal{F}_{D}^{\prime \prime \prime} u^{\prime \prime \prime}\right) u^{\prime}-3 u^{\prime \prime}\left(\mathcal{F}_{D}^{\prime \prime \prime} u^{\prime}-\mathcal{F}_{D}^{\prime \prime} u^{\prime \prime}\right)\right],
\end{aligned}
$$

where $^{\prime}=d / d a_{D}$ and $\mathcal{F}_{D}$ is the prepotential defined by

$$
a=\frac{d \mathcal{F}_{D}}{d a_{D}}
$$

and using (2.4) for $\Pi=a$ with inverse relation $d u / d a_{D}$ (see also Ref.17), we can arrive at the differential equation for $\mathcal{F}_{D}$

$$
\mathcal{F}_{D}^{(4)}+u^{\prime}\left(X-\frac{3 u^{\prime \prime}}{u^{\prime 2}}\right) \mathcal{F}_{D}^{\prime \prime \prime}=0
$$

This equation is integrated to give

$$
\mathcal{F}_{D}^{\prime \prime \prime}=c \frac{4 m^{2}-3 u}{\Delta} u^{\prime 3}
$$

where $c$ is an integration constant to be determined later. The factor $\left(4 m^{2}-3 u\right) / \Delta$ corresponds to the Wronskian $W$ defined in (2.7).

Since $\mathcal{F}_{D}^{\prime \prime \prime}$ manifestly vanishes at $u=4 m^{2} / 3, \mathcal{F}_{D}$ is represented by a linear combination of $a_{D}^{2}, a_{D}$ and 1 , although $O\left(a_{D}\right)$-terms can be neglected, thus it follows immediately from (3.13) that $a \propto a_{D}$. This induces the trivial monodromy

$$
\left(\begin{array}{c}
a \\
a_{D}
\end{array}\right) \longrightarrow\left(\begin{array}{ll}
1 & 0 \\
0 & 1
\end{array}\right)\left(\begin{array}{c}
a \\
a_{D}
\end{array}\right),
$$

which indicates that the indicial indices of the Picard-Fuchs equation at $u=4 m^{2} / 3$ are integers and the BPS spectrum is unchanged at this point.

Remark: The same monodromy can be seen from a version of (3.15) in weak coupling region.

As is easy to find that, only what we need is a function $u=u\left(a_{D}\right)$ to determine $\mathcal{F}_{D}$, but is obtainable from inverting the solution of the Picard-Fuchs equation. Therefore, substituting these data into (3.15) and triply integrate it, we will be able to determine $\mathcal{F}_{D}$. On the other 
hand, it would be sufficient to once determine $c$ at a representative point in the moduli space. Since $c$ has mass dimension zero, it can be regarded as a pure number. In fact, $c$ can be determined at the massless point and the result

$$
c=\frac{i}{16 \pi}
$$

follows from comparing (3.15) with the massless prepotential.21

The next one to be done is to calculate $u^{\prime}$, but is a easy task. Since $u^{\prime}=\left(d a_{D} / d u\right)^{-1}$, it is sufficient to obtain the solution to the Picard-Fuchs equation at $u=e_{1}$. With the help of (3.4), it is found by a linear combination

$$
\frac{d a_{D}}{d u}=\rho_{1} \varphi_{1}+\rho_{2} \varphi_{2}
$$

where

$$
\begin{aligned}
\varphi_{1}= & 1-\frac{6 u}{4 m^{2}-3 e_{1}}-\frac{u^{2}}{e_{1}\left(2 e_{1}-e_{2}\right)\left(2 e_{1}-e_{3}\right)\left(3 e_{1}-4 m^{2}\right)^{2}}\left[9 e_{1}^{2}\left(36 e_{1}-e_{2}-e_{3}\right)\right. \\
& \left.-12\left(112 e_{1}^{2}-3 e_{1} e_{2}-3 e_{1} e_{3}+e_{2} e_{3}\right) m^{2}+512\left(3 e_{1}-m^{2}\right) m^{4}+36 m\left(3 e_{1}-4 m^{2}\right) \Lambda^{3}\right]-\cdots, \\
\varphi_{2}= & u-\frac{3 e_{1}^{2}\left(4 e_{1}-e_{2}-e_{3}\right)-4\left(8 e_{1}^{2}-3 e_{1} e_{2}-3 e_{1} e_{3}+e_{2} e_{3}\right) m^{2}}{2 e_{1}\left(2 e_{1}-e_{2}\right)\left(2 e_{1}-e_{3}\right)\left(3 e_{1}-4 m^{2}\right)} u^{2}-\cdots .
\end{aligned}
$$

Since these $\varphi_{i}$ are extremely complicated functions in the language of explicit $e_{i}$, i.e., right hand sides of $e_{i}$ in (3.11), we do not try to express $\varphi_{i}$ by using them. The constants $\rho_{i}$ are determined from asymptotic expansion of the period integral of $a_{D}$, i.e.,

$$
\rho_{1}=-\frac{1}{2^{1 / 6} \sqrt{3} \Lambda}, \rho_{2}=-\frac{25}{9 \Lambda^{3}} \sqrt{\frac{2}{3}} .
$$

Integration produces $a_{D}$ itself with an integration constant, but from dimensional analysis, it is found that it has a unit mass dimension. Since all dependence of the scale parameter (instanton correction) should be entered in the right hand side of (3.18), the integration constant must be proportional to $\mathrm{m}$. In addition, since the massless theory does not have this term, it can be regarded as a characteristic feature in massive theory. Actually, it is a residue contribution from the massive meromorphic 1-form, and this is the case. Thus,

$$
a_{D}=2 \pi i n \operatorname{res}(\lambda)+\int_{0}\left(\rho_{1} \varphi_{1}+\rho_{2} \varphi_{2}\right) d u
$$

where $n \in \boldsymbol{Z}$ is the winding number of the $\beta$-cycle which loops around the pole of the massive meromorphic 1 -form and the residue is evaluated at $x=0$. Denoting $\widetilde{a}_{D}=a_{D}-2 \pi i n$ res $(\lambda)$ and repeatedly solving (3.21), one can arrive at the inverse relation $u=u\left(a_{D}\right)$. 
In this way, the result

$$
\begin{aligned}
\mathcal{F}_{D}= & \frac{1}{2} c_{1} \widetilde{a}_{D}^{2}+c_{2} \widetilde{a}_{D}+c_{3} \\
& -i \frac{\Lambda^{2}}{\pi} \frac{\widetilde{a}_{D}^{2}}{\left(e_{1}-e_{2}\right)\left(e_{1}-e_{3}\right)}\left[\frac{3}{2^{17 / 3}}\left(3 e_{1}-4 m^{2}\right)\left(\ln \widetilde{a}_{D}^{2}-3\right)+\frac{1}{2^{29 / 6} 3^{2 / 3}\left(e_{1}-e_{2}\right)\left(e_{1}-e_{3}\right)}\right. \\
& {\left[125\left(e_{1}-e_{2}\right)\left(e_{1}-e_{3}\right)\left(3 e_{1}-4 m^{2}\right)+9 \cdot 2^{7 / 3}\left(e_{2}+e_{3}-2 e_{1}\right) m^{2} \Lambda^{2}\right.} \\
& \left.\left.+27 \cdot 2^{1 / 3}\left(6 e_{1}^{2}+4 e_{2} e_{3}-5 e_{1}\left(e_{2}+e_{3}\right)\right) \Lambda^{2}\right] \frac{\widetilde{a}_{D}}{\Lambda}+\cdots\right]
\end{aligned}
$$

where $c_{i}$ are integration constants, follows from expanding (3.15). $c_{2} \widetilde{a}_{D}+c_{3}$ may be neglected because this terms does not change the effective coupling constant, but $c_{1}$ is non-trivial. Dimensional analysis shows that $c_{1}$ is a pure number. Therefore, it is sufficient to choose it as a constant such that $\mathcal{F}_{D}$ for $m \rightarrow 0$ coincides with that in the massless theory. As a check, one can see that this $\mathcal{F}_{D}$ reduces to that of massless theory for $m \rightarrow 0$, provided

$$
c_{1}=-\frac{i}{4 \pi} \ln \Lambda^{2}
$$

The reader might already noticed that actually this prepotential was obtained irrelevantly from details of $e_{1}$, although it was calculated under the assumption of small mass to see a connection with the massless theory. For general $m$ in strong coupling regime, only the differences of the final form of the prepotential are explicit value of $e_{i}, c_{i}$ and $c$. For instance, for a large mass case, it is enough to simply expand (3.6) near $m=\infty$ to get the zeros of $\Delta$ and replace $e_{i}$ by, e.g.,

$$
e_{1}=m^{2}+\frac{\Lambda^{3}}{8 m}+\cdots, e_{2}=\Lambda^{3 / 2} \sqrt{m}-\frac{\Lambda^{3}}{16 m}+\cdots, e_{3}=-\Lambda^{3 / 2} \sqrt{m}-\frac{\Lambda^{3}}{16 m}+\cdots
$$

The period $a_{D}$ can be obtained from simply substituting (3.24) into (3.18). In this way, the dual prepotential for a large mass is calculated, but it's form is not so attractive for us because it is again written by a complicated function in $e_{i}$. For this reason, it would not be necessary to write down the dual prepotential for $|m| \geqq 1$, and even for other $N_{f}$ cases.

\section{SUMMARY}

In this paper, it has been shown that the Wronskian of Picard-Fuchs equation in massive theory is related with (differentiation of) Matone's modular invariant through a differential 
equation. By comparing the solution to this equation with the Whitham dynamics, we have found a closed form of $\partial \mathcal{F} / \partial T_{0}$, which has not been evaluated so far. In this way, we have found a general form of the massive scaling relation. In the pure $\mathrm{SU}(2)$ theory, the scaling relation of prepotential is known to be also obtained as an anomalous superconformal Ward identity, 22 therefore, it would be a natural question to ask whether 2.25 ) can be obtained as "an anomalous superconformal Ward identity" in this $N_{f}=1$ massive theory.

On the other hand, we have also found that the differential equation for the Wronskian gives a differential relation between prepotential and moduli, which is quite reminiscent of the one presented in the five dimensional gauge theory 17 and the dual prepotential is calculated from this equation.

More detailed study of a relation between Wronskian and modular invariant of Matone will provide us more useful informations on the massive prepotentials also in the future.

\section{ACKNOWLEGMENT}

The author acknowledges Prof. K. Ito (YITP) for discussions on miscellaneous aspects of $N=2$ Seiberg-Witten models. 


\section{REFERENCES}

${ }^{1}$ N. Seiberg, Phys. Lett. B 206, 75 (1988).

${ }^{2}$ M. T. Grisaru and W. Siegel, Nucl. Phys. B 201, 292 (1982).

${ }^{3}$ P. S. Howe, K. S. Stelle and P. K. Townsend, Nucl. Phys. B 214, 519 (1983).

${ }^{4}$ P. S. Howe, K. S. Stelle and P. K. Townsend, Nucl. Phys. B 236, 125 (1984).

${ }^{5}$ P. S. Howe, K. S. Stelle and P. C. West, Phys. Lett. B 124, 55 (1983).

${ }^{6}$ N. Seiberg and E. Witten, Nucl. Phys. B 431, 484 (1994).

${ }^{7}$ N. Seiberg and E. Witten, Nucl. Phys. B 435, 129 (1994).

${ }^{8}$ A. Klemm, W. Lerche and S. Theisen, Int. J. Mod. Phys. A 11, 1929 (1996).

${ }^{9}$ Essays on mirror manifolds, edited by S. T. Yau (International Press, Hong Kong, 1992).

${ }^{10}$ P. Candelas, X. C. de la Ossa, P. S. Green and L. Parkes, Nucl. Phys. B 359, 21 (1991).

${ }^{11}$ P. Candelas, X. C. de la Ossa, P. S. Green and L. Parkes, Phys. Lett. B 258, 118 (1991).

${ }^{12}$ M. Matone, Phys. Lett. B 357, 342 (1995).

${ }^{13}$ J. Sonnenschein, S. Theisen and S. Yankielowicz, Phys. Lett. B 367, 145 (1996).

${ }^{14}$ T. Eguchi and S.-K. Yang, Mod. Phys. Lett. A 11, 131 (1996).

${ }^{15}$ A. Gorsky, I. Krichever, A. Marshakov, A. Mironov and A. Morozov, Phys. Lett. B 355, 466 (1995).

${ }^{16}$ T. Nakatsu and K. Takasaki, Mod. Phys. Lett. A 11, 157 (1996).

${ }^{17}$ H. Kanno and Y. Ohta, Nucl. Phys. B 530, 73 (1998).

${ }^{18}$ K. Ito and S.-K. Yang, Phys. Lett. B 366, 165 (1996).

${ }^{19}$ A. Hanany and Y. Oz, Nucl. Phys. B 452, 283 (1995).

${ }^{20}$ Y. Ohta, J. Math. Phys. 37, 6074 (1996).

${ }^{21}$ K. Ito and S.-K. Yang, "Picard-Fuchs equations and prepotentials in $N=2$ supersym- 
metric QCD," hep-th/9603073.

${ }^{22}$ P. S. Howe and P. C. West, Nucl. Phys. B 486, 425 (1997). 